\title{
A Review of Long-Term Mechanical Circulatory Support as Destination Therapy: Evolving Paradigms for Treatment of Advanced Heart Failure
}

\author{
Ali A. Valika, ${ }^{1}$ and William Cotts ${ }^{2}$ \\ ${ }^{1}$ Midwest Heart Foundation, 133 E. Brush Hill Road, Suite 202, Elmhurst, IL 60126, USA \\ ${ }^{2}$ Heart Transplantation and Mechanical Assistance, Advocate Christ Medical Center, 4440 W. 95th Street, Suite 407, Oak Lawn, \\ IL 60453, USA \\ Correspondence should be addressed to William Cotts; wcotts@nmff.org
}

Received 16 October 2012; Accepted 1 November 2012

Academic Editors: J. C. Chachques and J. Lavee

Copyright (C) 2013 A. A. Valika and W. Cotts. This is an open access article distributed under the Creative Commons Attribution License, which permits unrestricted use, distribution, and reproduction in any medium, provided the original work is properly cited.

Left ventricular assist devices as long-term mechanical circulatory support are increasingly utilized as an option for medically refractory advanced heart failure. Rapid advances in this field, from pulsatile paracorporeal flow pumps to now more advanced intracorporeal continuous flow devices, have led to more wide spread use of device therapy. Several trials have now confirmed the survival benefits of ventricular assist devices, not only as a method for bridging patients waiting on the transplant list, but also as an evolving paradigm of destination therapy. Significant improvements in quality of life and functional status have been reported in patients receiving these devices. Survival outcomes with this therapy continue to improve, and long term durability of newer generation devices remains yet to be discerned. Comparative data to heart transplantation remains scarce. This paper will focus on the historical development of ventricular assist device therapy for advanced heart failure, review major trials of destination therapy, and look at comparative literature in the modern era to cardiac transplantation.

\section{Introduction}

Left ventricular assist devices (LVAD) as long term mechanical circulatory support (MCS) therapy are being used with increasing frequency for medically refractory heart failure. Over 4000 patients in the United States have received LVAD implants, and this number continues to grow [1]. Technological advancements have rapidly advanced this field, with the replacement of pulsatile paracorporeal flow pumps with intracorporeal continuous flow devices. Several trials have now confirmed the survival benefits of LVAD therapy, not only as a method for bridging patients waiting on the transplant list, but also as an evolving paradigm of destination therapy (DT) [2]. Significant improvements in quality of life and functional status have now been reported in patients receiving these devices [3]. This review will focus on the historical development of LVADs, review major trials of LVAD use as destination therapy, and look at comparative literature in the modern era to cardiac transplantation.

\section{History}

The development of MCS support has evolved over time. Work on engineering designs on LVAD therapy as long term support began in the late 1960s, and after several years of trialed designs, the first HeartMate IP LVAS system was approved for use in the United States in 1994. The first generation LVADs produced pulsatile flow and simulated cyclic stroke volume with normal physiologic blood pressure and pulse. Among these devices, the WorldHeart Novacor and Thoratec HeartMate XVE were initially trialed for use as DT. Several complications limited the long-term use of these pulsatile devices, including high rates of thromboembolic events, driveline and bloodstream infections, bleeding, and ultimately device failure [4]. This latter complication limited the longevity of device therapy in patients for use as DT and was thought to be related to the nondurability of mechanical bearings that allowed for pulsatile flow to occur. In fact, device malfunction was noted in up to 
$35 \%$ of patients in study trials, occurring approximately 18-24 months after implant [4]. These limitations led to the conceptual development of continuous flow technology, to minimize bearing wear and improve longevity of device mechanics. A rapid increase in device implants began to occur with the use of continuous flow LVAD technology. The Food and Drug Administration (FDA) granted approval of the first continuous flow LVAD (HeartMate II) in 2008 as a bridge to transplant (BTT), and subsequently as DT in January 2010. The development of the Interagency Registry for Mechanically Assisted Circulatory Support (INTERMACS) began documenting LVAD implants within a database since June 23, 2006. Since that time, over 4,000 patients have been entered into the database with a primary device implant [1]. By the mid of year 2011, more than $99 \%$ of those LVAD implants were continuous flow devices [1].

\section{Destination Therapy Trials}

The first DT trial, published in 2001, was the Randomized Evaluation of Mechanical Assistance for the Treatment of Congestive Heart Failure (REMATCH) trial, which paved the way for future studies in this arena. This multicenter study enrolled 129 patients between May 1998 and July 2001 with end-stage heart failure, who were deemed ineligible for heart transplant, to either implantation of HeartMate VE LVAD (pulsatile flow device) or optimal medical management. Entry criteria included New York Heart Association (NYHA) class IV heart failure, left ventricular ejection fraction $<25 \%$, and either peak oxygen consumption $<12 \mathrm{~mL} / \mathrm{kg} / \mathrm{min}$ or intravenous inotropic therapy [4]. All patients were estimated to have a life expectancy of less than two years. The trial demonstrated a marked survival benefit. At 1 year, LVAD therapy revealed a superior survival rate compared to optimal medical therapy (52\% versus $23 \%, P=0.002$ ), and this benefit continued at 2 years $(23 \%$ versus $8 \%, P=0.009)$. There was a 48 percent reduction in the risk of all-cause death in the group that received LVAD, as compared with the medical-therapy group (relative risk, 0.52; 95 percent confidence interval, 0.34 to $0.78 ; P=0.001)$. Significant improvement in functional NYHA class was noted at 1 year of study followup $(P<0.001)$, and quality of life as assessed by the 36-item Medical Outcomes Study Short-Form General Health Survey (SF-36) was also significantly improved at 1 year as compared to medical therapy $(P=0.01)$. However, the frequency of adverse events in the device group was twofold greater than in the medical-therapy group, with a predominance of infection, bleeding, and device malfunction [4].

Several points can be gleaned from this landmark trial. First, survival, as well as quality of life, was improved by the use of LVAD therapy as compared to medical therapy. However, despite optimal care, mortality was still quite dismal in both arms of the study, suggesting the severity of end-stage heart failure at entry into the study, with the majority of patients requiring intravenous inotropic support. The dismal survival of $25 \%$ at 2 years in the LVAD arm was still quite alarming, and multiple factors may have contributed to this high mortality. Given the mechanics of first generation devices, the high rate of device malfunction (35\%) certainly was a causal factor, along with infection, bleeding, ischemic stroke, and a noted learning curve for device implantation and patient selection [4]. Despite the suboptimal survival in this trial, a new era of device therapy had now been introduced to a cohort of patients with very limited medical options, thus paving the way for future developments in device mechanics.

Looking further at improvements in patient selection, post-REMATCH era implant survival for DT therapy continued to improve. In a study of 280 patients with HeartMate XVE implants for DT subsequent to the REMATCH trial, the 1-year survival after LVAD implantation improved slightly to $56 \%$. However, stratification of DT candidates by risk score assessment revealed that, particularly in the low risk cohort, survival could be as high as $81 \%$ at 1 year. The main causes of death appeared to be sepsis, right heart failure, and multiorgan failure, with preoperative worsening endorgan function as a marker for worse outcomes after LVAD implant [5]. This provided further evidence of the importance of careful patient selection for LVAD implantation.

The Clinical Utility Baseline Study (CUBS) trial was the first European study of DT that evaluated the LionHeart-2000 (LionHeart Ventricular Assist System), as a fully implantable LVAD. The device was unique in its ability to be powered by transcutaneous energy transfer, thereby eliminating the need for external drive lines, and decreasing the potential for infection. This was a small, nonrandomized, observational trial, with only 23 patients enrolled in the study between October 1999 and December 2002. All patients were in NYHA class IV heart failure and were deemed ineligible for heart transplantation. As predicted, the LionHeart device caused less infectious complications in comparison to the REMATCH data cohort. However, the survival rates in the patients receiving the device were markedly inferior (1year survival 39\%, 2-year survival 22\%) compared to prior REMATCH data. This increased mortality was thought once again to be secondary to enrollment of higher risk patients, who may have had excessive operative risk at the time of implant [6]. The DT strategy once again appeared to be limited by device complications and a high mortality rate in this study population.

The Investigation of Nontransplant-Eligible Patients Who Are Inotrope Dependent (INTREPID) trial results were revealed in 2007. This multicenter trial looked at the use of the Novacor LVAD device in 55 patients enrolled between 2001 and 2003. 37 patients underwent LVAD implantation and 18 remained on medical management. The LVAD-treated patients had superior survival rates compared to medical treatment at 6 months ( $46 \%$ versus $22 \% ; P=0.03)$ and 12 months (27\% versus $11 \% ; P=0.02)$. Although overall survival was improved, the Nocavor LVAD outcomes were inferior to those of the HeartMate VE in the REMATCH trial. Again, patient selection may have played a role in the high mortality noted. However, device design and antithrombotic strategies may have also contributed, as $62 \%$ of LVAD patients experienced a stroke or transient ischemic attack during the study, contributing to the highest cause of death (34\%) during 
the trial [7]. Since then, improvements in implant technique and anticoagulation strategies have improved the stroke risk with this particular device [8]. However, the pulsatile devices, as a group, appeared continually to be inferior in device durability and longevity, limiting ultimate long term utility as a DT strategy.

A major shift in paradigm for DT began as continuous flow technology reached the clinical trial arena. The HeartMate II continuous flow LVAD system was designed as a smaller pump for implant feasibility with a solitary rotary component, allowing for axial flow through the device with limited wearing of mechanical parts. This increased the longevity of device mechanics and yet also provided an interesting change in patient physiology, that of pulseless continuous blood flow to the systemic circulation. The HeartMate II investigators published their randomized trial data in 2009, comparing the pulsatile HeartMate XVE versus the continuous flow HeartMate II device. They enrolled 200 patients from March 2005 to May 2007, who met the following criteria: a left ventricular ejection fraction of less than $25 \%$; a peak oxygen consumption of less than $14 \mathrm{~mL} / \mathrm{kg} / \mathrm{min}$; NYHA class IIIB or IV symptoms for at least 45 of 60 days, or dependence on an intra-aortic balloon pump for 7 days, or inotropes for at least 14 days before enrollment. The results were remarkable in that actuarial survival estimates were substantially improved with the HeartMate II device in comparison to the Heartmate XVE device (68\% versus $55 \%$ at 1 year, $58 \%$ versus $24 \%$ at 2 years, $P=0.008$ ). Also, significant reductions in the rates of major adverse events were noted with continuous-flow LVAD technology, including a decrease in infection rates, right heart failure, renal failure, and cardiac arrhythmia. There was a $38 \%$ relative risk reduction in the rate of rehospitalization among patients with the HeartMate II as compared with the HeartMate XVE device implant [9].

To further this marked advance in survival with new generation technology, the HeartMate II investigators recently published a review of 281 patients, with similar entry criteria, who were implanted from May 2007 to March 2009, and compared these patients with the initial study cohort as noted above. Compared with the early trial group, patients in this cohort had further reduced adverse event rates for bleeding (1.66 versus 1.13 events per patient-year, $P<0.001$ ), sepsis ( 0.38 versus $0.27, P=0.025)$, device-related infections $(0.47$ versus $0.27, P<0.001)$, and hemorrhagic stroke $(0.07$ versus $0.03, P=0.01)$. There was a trend towards increased survival at 1 year in the more recent cohort compared to the early trial group (73\% versus $68 \%, P=0.21)$ [10]. As familiarity and experience continued to advance with the second generation devices, patient outcomes similarly improved.

In the more recent experience of continuous flow devices, actuarial survival at 1 year since implant now approximates $80 \%$ within the INTERMACS registry and only marginally diminishes to $69 \%$ at 2 years [1]. Survival notably continues to improve within the more recent era of LVAD technology, and improvements in patient selection have noted a decrease in implants particularly in the INTERMACS 1 category of patients, that of critical cardiogenic shock. The strategy of DT continues to evolve in the United States, with a notable shifting paradigm. The percentage of implants with a BTT strategy decreased from $44 \%$ in 2006 down to $23 \%$ in 2011. Reciprocally, a twofold increase in the proportion of implants for DT has been noted (16\% in 2006 versus 34\% in 2011). No further pulsatile devices have been implanted since 2010 as a DT strategy [1].

\section{Comparison to Heart Transplantation}

Given the marked advances within the last decade in MCS, with the noted increase in survival offered by current LVAD technology, what can be said about comparative data to cardiac transplantation? One-year survival for cardiac transplantation approximates $85 \%$ in the era of 2002 to 2008 , with the highest mortality occurring within the first 6-months. Improved immunosuppression strategies have particularly enhanced this initial 6 month survival. However, the subsequent median survival of 10-13 years after transplant has not substantially changed over the last decade of cardiac transplantation [11]. Long term survival and durability of the continuous flow devices as DT therapy have yet to be born out, with case reports of event-free survival over four years in certain device implants [12-14]. Improvements in peak oxygen consumption occur similarly in both cardiac transplantation and LVAD assist therapy [15]. In a study comparing extended criteria donor heart recipients versus those of LVAD implants as a DT strategy, similar postoperative and 1-year mortality has been noted in both groups, though 3-year survival outcomes were still better with transplantation [16]. Quality of life as assessed by selfreported questionnaires appears also to be slightly improved with cardiac transplantation versus that of LVAD therapy, acknowledging the differences in lifestyles to be had with driveline and battery management versus that of medication adherence to an immunosuppression regimen [17]. In studies of cost effectiveness comparing transplant data with LVAD implants, total actuarial hospital costs for LVADs implanted in the era of pulsatile devices (1996-2000) exceeded that of cardiac transplantation $(\$ 197,957 \pm 77,291$ for LVAD versus $\$ 151,646 \pm 53,909$ for transplant, $P=0.005$ ) [18]. However, continuous flow devices have since markedly diminished the costs of implants, with more recent studies suggesting a $75 \%$ reduction in the incremental cost-effectiveness ratio as compared to the pulsatile device era $(\$ 198,184$ per qualityadjusted life year for continuous flow devices versus $\$ 802,700$ per quality-adjusted life year for pulsatile devices) [19]. In the current era, it will be difficult to assess overall comparisons, until one has an appropriate matched patient cohort with similar length of followup for both LVAD therapy and heart transplantation. Donor selection will continue to limit the availability of heart transplants, whereas institutional resources, costs of care, and adverse events associated with device therapy in the current era will continue to limit its durability and widespread utility [20].

\section{Current Guidelines}

Current guidelines among various heart failure societies have now incorporated destination therapy as part of an acknowledged treatment algorithm for patients with advanced heart 
failure. The 2009 update to the American College of Cardiology/American Heart Association guidelines for heart failure management recommend consideration of an LVAD as destination therapy as reasonable in highly selected patients with refractory end-stage heart failure and estimated oneyear mortality over 50 percent with medical therapy [21]. The 2010 Heart Failure Society of America guidelines recommend destination therapy with a permanent mechanical assist device in highly selected patients with severe HF refractory to conventional therapy who are not candidates for heart transplantation, particularly those who cannot be weaned from intravenous inotropic support at an experienced heart failure center [22]. The 2012 European Society of Cardiology guidelines offer a Class IIA indication for LVAD therapy in patients who are not transplant eligible as a DT strategy but also give credence to the fact that earlier implantation in less severely ill patients, before right ventricular or multiorgan failure develops, may lead to better surgical outcomes [23].

\section{Future Directions}

LVAD technology continues to rapidly advance, and new investigational devices continue to improve on prior designs. Newer third generation devices continue to be smaller in size and weight, with improved ease of implantation. The Heartware LVAD, as an example, is a smaller size continuous flow pump with centrifugal flow design, with a magnetically levitated impeller to allow for contact-free rotation, which is implanted completely within the pericardial space, minimizing the need for an abdominal pocket. This device has been commercially available in Europe for several years now and is under current FDA review for approval as a DT strategy in the US [24]. Other third generation devices utilizing magnetic based levitation in the horizon include the HeartMate III, the Berlin Heart Incor, and the DuraHeart [25]. Total artificial heart technology is slowly emerging, with a benefit particularly in those patients with biventricular failure who may not be LVAD candidates [26, 27]. Advances in device therapies for right ventricular failure, for indications in both the perioperative setting and for long term destination use, have been developed with newer right ventricular assist devices in the horizon [28]. Ultimately, fully implantable LVAD technology will likely reach clinical trials in the near future and then pave the way for further paradigm shifts in advanced heart failure management.

\section{References}

[1] J. K. Kirklin, D. C. Naftel, R. L. Kormos et al., "The Fourth INTERMACS Annual Report: 4, 000 implants and counting," The Journal of Heart and Lung Transplantation, vol. 31, no. 2, pp. 117-126, 2012.

[2] J. C. Fang, "Rise of the machines-left ventricular assist devices as permanent therapy for advanced heart failure," The New England Journal of Medicine, vol. 361, no. 23, pp. 2282-2285, 2009.

[3] J. G. Rogers, K. D. Aaronson, A. J. Boyle et al., "Continuous flow left ventricular assist device improves functional capacity and quality of life of advanced heart failure patients," Journal of the
American College of Cardiology, vol. 55, no. 17, pp. 1826-1834, 2010.

[4] E. A. Rose, A. C. Gelijns, A. J. Moskowitz et al., "Long-term use of a left ventricular assist device for end-stage heart failure," The New England Journal of Medicine, vol. 345, no. 20, pp. 1435-1443, 2001.

[5] K. Lietz, J. W. Long, A. G. Kfoury et al., "Outcomes of left ventricular assist device implantation as destination therapy in the post-REMATCH era: implications for patient selection," Circulation, vol. 116, no. 5, pp. 497-505, 2007.

[6] W. E. Pae, J. M. Connell, A. Adelowo et al., "Does total implantability reduce infection with the use of a left ventricular assist device? The lion heart experience in Europe," Journal of Heart and Lung Transplantation, vol. 26, no. 3, pp. 219-229, 2007.

[7] J. G. Rogers, J. Butler, S. L. Lansman et al., "Chronic mechanical circulatory support for Inotrope-dependent heart failure patients who are not transplant candidates. Results of the INTrEPID Trial," Journal of the American College of Cardiology, vol. 50, no. 8, pp. 741-747, 2007.

[8] J. T. Strauch, D. Spielvogel, P. L. Haldenwang et al., "Recent improvements in outcome with the novacor left ventricular assist device," Journal of Heart and Lung Transplantation, vol. 22, no. 6, pp. 674-680, 2003.

[9] M. S. Slaughter, J. G. Rogers, C. A. Milano et al., "Advanced heart failure treated with continuous-flow left ventricular assist device," The New England Journal of Medicine, vol. 361, no. 23, pp. 2241-2251, 2009.

[10] S. J. Park, C. A. Milano, A. J. Tatooles et al., "Outcomes in advanced heart failure patients with left ventricular assist devices for destination therapy," Circulation, vol. 5, no. 2, pp. 241-248, 2012.

[11] J. Stehlik, L. B. Edwards, A. Y. Kucheryavaya et al., "The Registry of the International Society for Heart and Lung Transplantation: twenty-seventh official adult heart transplant report 2010," Journal of Heart and Lung Transplantation, vol. 29, no. 10, pp. 1089-1103, 2010.

[12] J. M. Rogers, Are LVADs Ready to be Mainstream?vol. 11, Paradigm: Advances in Heart Failure Technologies, 2011.

[13] E. V. Potapov, Y. Weng, T. Drews, M. Jurmann, and R. Hetzer, "Longest time of support by the Novacor left ventricular assist device without pump exchange," Annals of Thoracic Surgery, vol. 80, no. 6, p. 2421, 2005.

[14] R. John, F. D. Pagani, Y. Naka et al., "Post-cardiac transplant survival after support with a continuous-flow left ventricular assist device: Impact of duration of left ventricular assist device support and other variables," Journal of Thoracic and Cardiovascular Surgery, vol. 140, no. 1, pp. 174-181, 2010.

[15] N. de Jonge, H. Kirkels, J. R. Lahpor et al., "Exercise performance in patients with end-stage heart failure after implantation of a left ventricular assist device and after heart transplantation: an outlook for permanent assisting?" Journal of the American College of Cardiology, vol. 37, no. 7, pp. 1794-1799, 2001.

[16] M. A. Daneshmand, K. Rajagopal, B. Lima et al., "Left ventricular assist device destination therapy versus extended criteria cardiac transplant," Annals of Thoracic Surgery, vol. 89, no. 4, pp. 1205-1210, 2010.

[17] K. L. Grady, P. M. Meyer, D. Dressler et al., "Change in quality of life from after left ventricular assist device implantation to after heart transplantation," Journal of Heart and Lung Transplantation, vol. 22, no. 11, pp. 1254-1267, 2003. 
[18] P. L. DiGiorgi, M. S. Reel, B. Thornton, E. Burton, Y. Naka, and M. C. Oz, "Heart transplant and left ventricular assist device costs," Journal of Heart and Lung Transplantation, vol. 24, no. 2, pp. 200-204, 2005.

[19] J. G. Rogers, R. R. Bostic, K. B. Tong, R. Adamson, M. Russo, and M. S. Slaughter, "Cost-effectiveness analysis of continuousflow left ventricular assist devices as destination therapy," Circulation, vol. 5, no. 1, pp. 10-16, 2012.

[20] K. Lietz and L. W. Miller, "Will left-ventricular assist device therapy replace heart transplantation in the foreseeable future?" Current Opinion in Cardiology, vol. 20, no. 2, pp. 132-137, 2005.

[21] S. A. Hunt, "2009 focused update incorporated into the ACC/AHA 2005 guidelines for the diagnosis and management of heart failure in adults: a report of the American college of cardiology foundation/American heart association task force on practice guidelines," Circulation, vol. 119, no. 14, pp. e391-e479, 2009.

[22] J. Lindenfeld, N. M. Albert, J. P. Boehmer et al., "HFSA 2010 comprehensive heart failure practice guideline," Journal of Cardiac Failure, vol. 16, no. 6, pp. e1-e2, 2010.

[23] J. J. McMurray, S. Adamopoulos, S. D. Anker et al., "ESC Guidelines for the diagnosis and treatment of acute and chronic heart failure 2012: the Task Force for the Diagnosis and Treatment of Acute and Chronic Heart Failure 2012 of the European Society of Cardiology. Developed in collaboration with the Heart Failure Association (HFA) of the ESC," European Heart Journal, vol. 14, no. 8, pp. 803-869, 2012.

[24] K. D. Aaronson, M. S. Slaughter, L. W. Miller et al., "Use of an intrapericardial, continuous flow, centrifugal pump in patients awaiting heart transplantation," Circulation, vol. 125, no. 25, pp. 3191-3200, 2012.

[25] J. Garbade, H. B. Bittner, M. J. Barten, and F.-W. Mohr, "Current trends in implantable left ventricular assist devices," Cardiology Research and Practice, vol. 2011, Article ID 290561, 9 pages, 2011.

[26] L. E. Samuels and R. Dowling, "Total artificial heart: destination therapy," Cardiology Clinics, vol. 21, no. 1, pp. 115-118, 2003.

[27] J. G. Copeland, R. G. Smith, F. A. Arabia et al., "Cardiac replacement with a total artificial heart as a bridge to transplantation," The New England Journal of Medicine, vol. 351, no. 9, pp. 859-867, 2004.

[28] P. L. Hsu, J. Parker, C. Egger, R. Autschbach, T. Schmitz-Rode, and U. Steinseifer, "Mechanical circulatory support for right heart failure: current technology and future outlook," Artificial Organs, vol. 36, no. 4, pp. 332-347, 2012. 


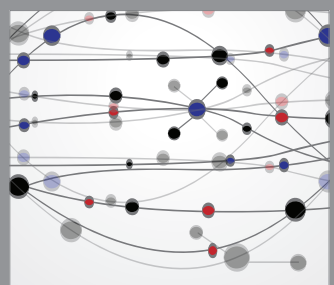

The Scientific World Journal
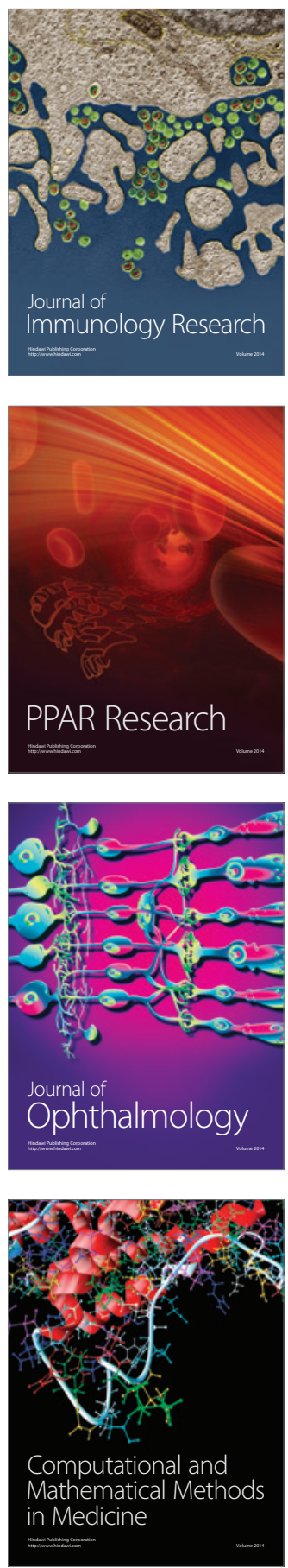

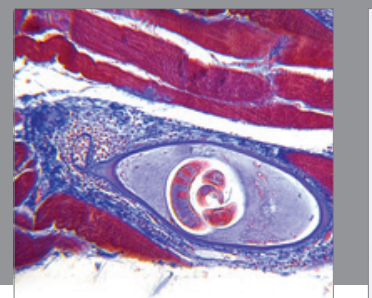

Gastroenterology

Research and Practice
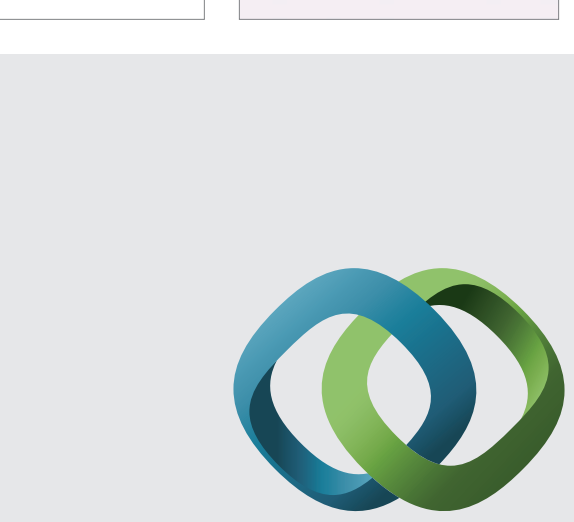

\section{Hindawi}

Submit your manuscripts at

http://www.hindawi.com
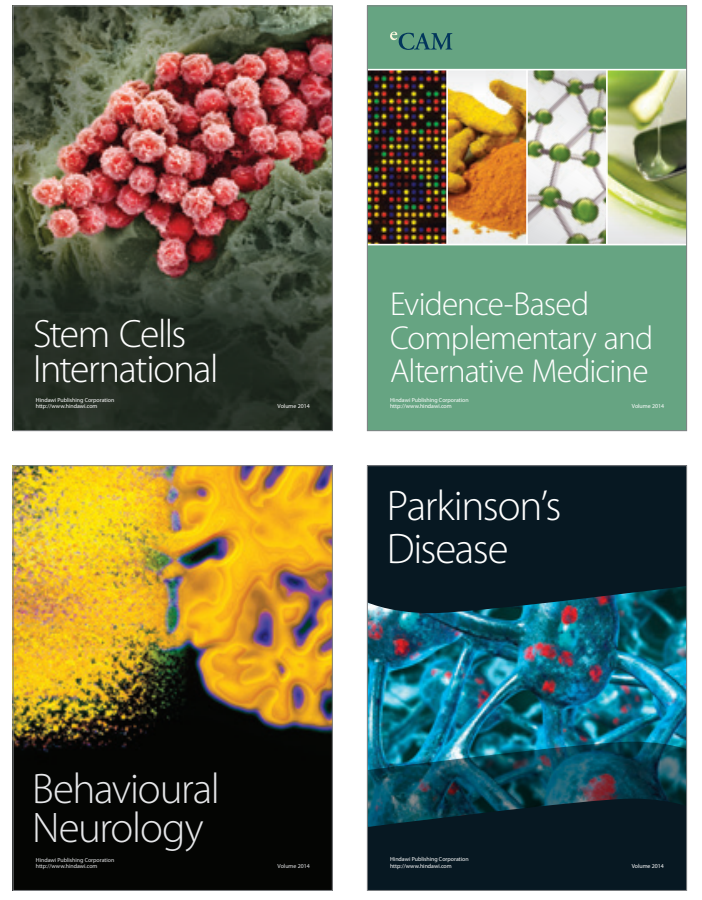
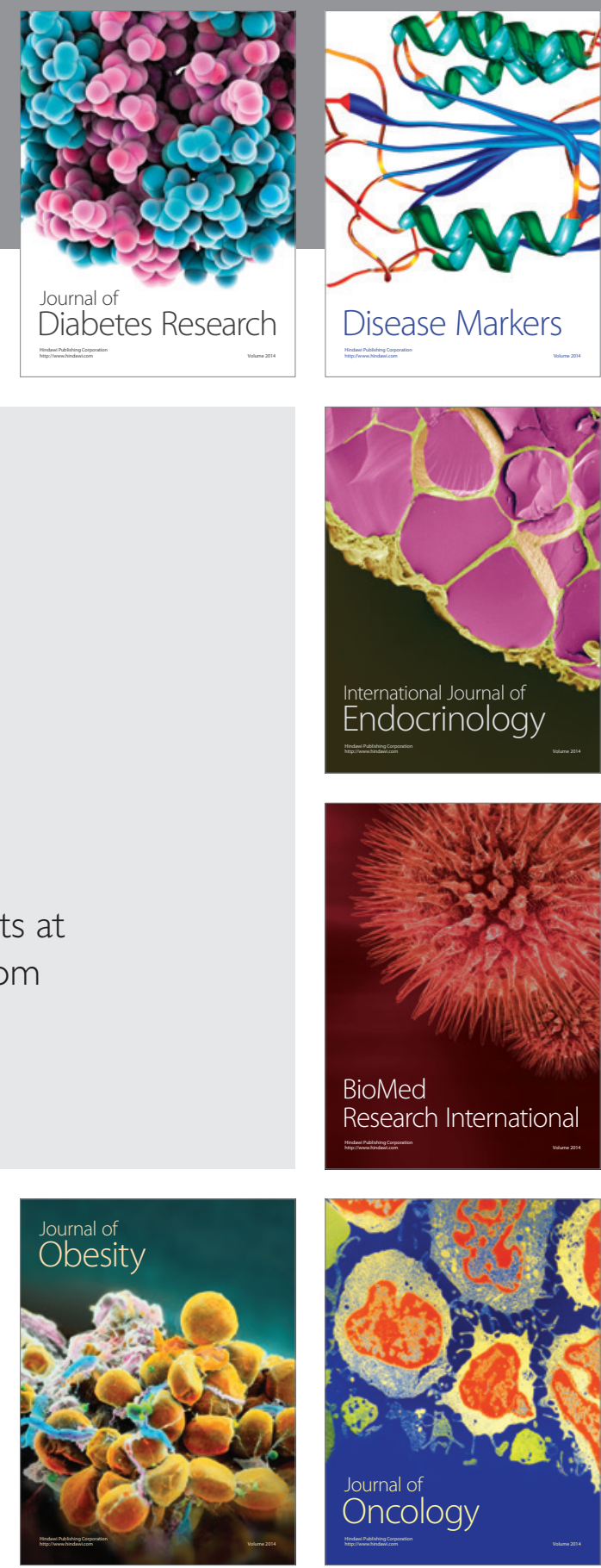

Disease Markers
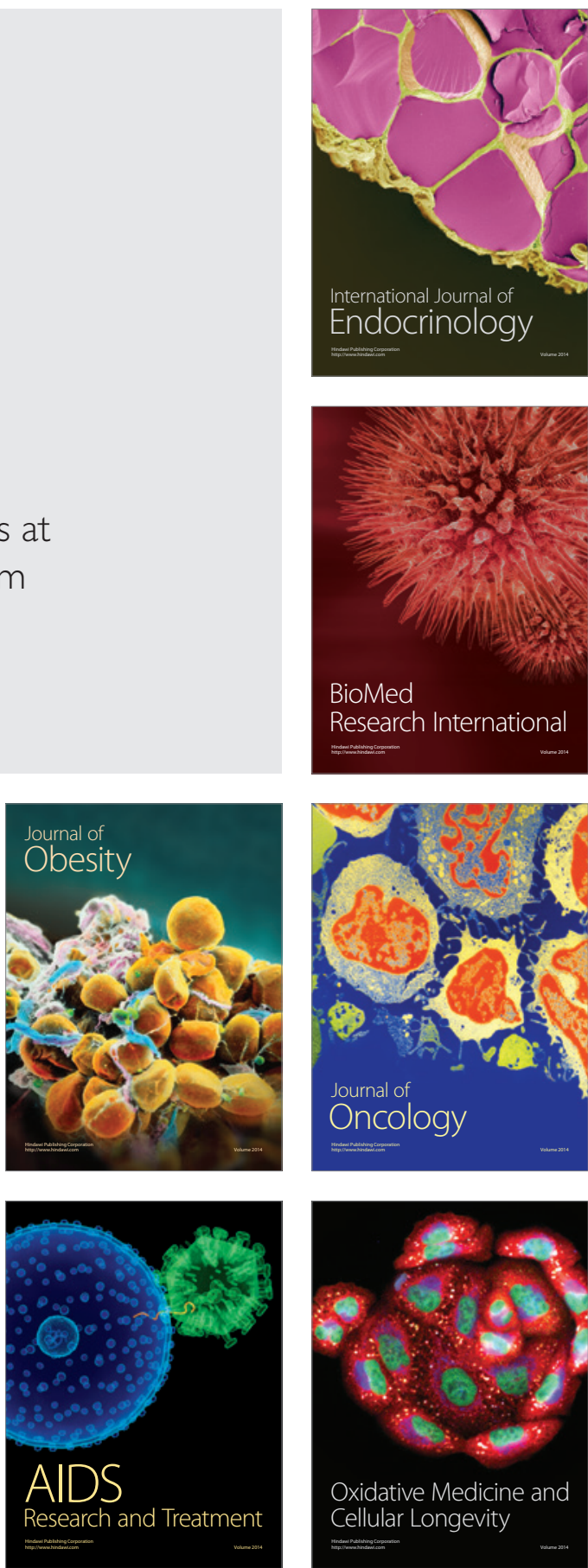